\title{
Possíveis influências na sexualidade de universitários da área de saúde
}

\author{
Possible influences on sexuality among healthcare university
}

\section{Posibles influencias sobre la sexualidad de universitarios del área de salud}

Recebido: 20/09/2013

Aprovado: 03/02/2014

\author{
Rodrigo Eurípedes da Silveira ${ }^{1}$ \\ Álvaro da Silva Santos ${ }^{2}$ \\ Maritza Rodrigues Borges ${ }^{3}$
}

0 presente estudo tem como objetivo descrever as possíveis influências de bebidas alcoólicas, religião, pais e universidade na sexualidade e a satisfação com aspectos de vida de ingressantes em cursos de graduação da área da saúde. De corte descritivo e exploratório e com amostragem de conveniência, foram incluídos 786 estudantes, dos quais 79,3\% eram do sexo feminino, com idade média de 19,2 $\pm 3,1$ anos. Para os acadêmicos, o uso de bebidas alcoólicas $(96,6 \%)$ e o ingresso na universidade $(50,4 \%)$ estimulavam a sexualidade e $54,3 \%$ dos jovens estavam satisfeitos com a universidade. Questiona-se a influência da universidade neste contexto e, as implicações nas condutas de saúde dos estudantes em sua prática futura. Descritores: Sexualidade; Adolescente; Satisfação pessoal; Estudantes de Ciências da Saúde.

This study aim to describe the possible influences of binge drinking, religion, parents and University in sexuality and the satisfaction with aspects of life for freshmen in graduate courses of the health area. With descriptive and exploratory and convenience cutting sample, were included 786 students, $79.3 \%$ of which were female, with an average age of $19.2 \pm 3.1$ years. For academics, the use of alcoholic beverages (96.6\%) and University (50.4\%) stimulated the sexuality and $54.3 \%$ of the young were satisfied with the University. If questionated the influence of the University in this context and the implications of health behaviors of students in their future practice.

Descriptors: Sexuality; Adolescent; Personal satisfaction; Students health occupations.

El presente estudio tiene como objetivo describir las posibles influencias del alcohol, la religión, los padres y la Universidad en la sexualidad y satisfacción con los aspectos de la vida para los estudiantes en cursos superiores del área de la salud. Con trazo descriptivo y exploratorio y muestra de conveniencia fueran incluidos 786 estudiantes, $79.3 \%$ de los cuales

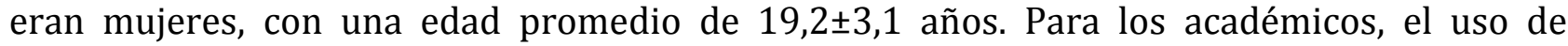
bebidas alcohólicas (96,6\%) y la Universidad (50.4\%) estimulan la sexualidad y 54,3\% de los jóvenes se mostraron satisfechos con la Universidad. Se cuestiona el papel de la Universidad en este contexto y las implicaciones en los comportamientos de salud de los estudiantes en su práctica futura.

Descriptores: Sexualidad; Adolescente; Satisfacción personal; Estudiantes del área de la salud.

\footnotetext{
${ }^{1}$ Enfermeiro. Mestre em Ciências da Saúde. Doutorando em Ciências pela Coordenadoria de Controle de Doenças da Secretaria de Estado de Saúde de São Paulo (SES-SP). rodrigoeuripedes.silveira@gmail.com.

${ }^{2}$ Enfermeiro. Especialista em Saúde Pública. Doutor em Ciências Sociais. Pós-Doutor em Serviço Social. Professor Adjunto III na Graduação em Enfermagem e da Pós-Graduação stricto sensu em Atenção à Saúde da Universidade Federal do Triângulo Mineiro (UFTM). alvaroenf@hotmail.com

${ }^{3}$ Enfermeira. Especialista em Enfermagem Obstétrica. Especialista em Acupuntura. Mestranda em Ciências da Saúde pelo Instituto de Assistência Médica ao Servidor Público Estadual de São Paulo (IAMSPE). francis.maritza@gmail.com
} 


\section{INTRODUÇÃO}

A sexualidade, concebida enquanto aspecto central e inerente à natureza humana engloba uma série de fatores como sexo, identidade de gênero, intimidade e reprodução. Tais aspectos ganham maior relevância e intensidade no período da adolescência, em que o jovem experimenta uma série de interações sociais, além da busca por sua autonomia e pela afirmação de sua identidade de gênero e suas funções, erotismo, prazer, intimidade e reprodução ${ }^{1,2}$.

Por muitas vezes, $\mathrm{o}$ adolescente tem a oportunidade de ingressar na Universidade, sobretudo no Brasil, em que o curso superior é o caminho natural da formação, especialmente na cultura das classes média e alta, além de ser um caminho privilegiado para a ascensão social e realização profissional $^{3}$.

Neste contexto, acontece a "fusão" do viver acadêmico com as características mais efusivas da adolescência, confluindo em situações e comportamentos que oferecem riscos e vulnerabilidades a estes jovens, a exemplo do tabagismo, o consumo excessivo de bebidas alcoólicas, a conduta sexual de risco, o uso de drogas ilícitas e outros 4 .

As Doenças Sexualmente Transmissíveis (DSTs), por sua vez, na América Latina, vitimam cerca de 1,4 milhões de pessoas infectadas pelo vírus da Imunodeficiência Humana (HIV), sendo que metade deste número são de brasileiros ${ }^{5,6}$. Entre as hipóteses, considera-se o consumo de álcool na adolescência ${ }^{1}$ como fator predisponente ao comportamento sexual de risco, sobretudo nas várias oportunidades de eventos sociais oportunizados e justificados pelo ingresso universitário, somado a fatores como a distância dos pais/responsáveis, a experiência de morar sozinho ou em repúblicas/pensionatos com um grupo de amigos, entre outros ${ }^{4}$.

Algumas investigações têm abordado aspectos relevantes sobre a temática, sobretudo acerca da percepção de risco pessoal e a conduta preventiva, cujos resultados apontam elevado conhecimento sobre os riscos inerentes a uma conduta sexual desprotegida a despeito da nãoredução dessas práticas ${ }^{7,8}$.

Embora estudantes da área de saúde mantenham amplo acesso às informações na universidade e a utilizem na abordagem à clientela através de orientações, diagnósticos e tratamentos, estudos tem demonstrado que seu nível de conhecimento sobre DST/AIDS não reduzem suas próprias atividades sexuais de risco ${ }^{5,8,9}$, por talvez se considerarem invulneráveis a adquirir tais doenças.

Esta investigação tem como objetivo compreender quais as possíveis influências de fatores como religião, ingresso na universidade e consumo de bebidas alcoólicas influenciarem na satisfação e no comportamento sexual de estudantes universitários da área de saúde.

\section{MÉTODO}

Trata-se de um estudo de delineamento descritivo, com recorte transversal e amostragem de conveniência, em que foram incluídos estudantes dos primeiros períodos dos cursos de graduação em Biomedicina, Enfermagem, Fisioterapia, Nutrição, Medicina, Psicologia e Terapia Ocupacional, que se matricularam nos anos de 2011 e 2012 na Universidade Federal do Triângulo Mineiro (UFTM). A coleta de dados ocorreu entre março de 2011 e novembro de 2012.

Utilizou-se um questionário semiestruturado, testado e ajustado em teste piloto em outra instituição de ensino superior, com aplicação supervisionada. Este estudo foi avaliado e aprovado pelo Comitê de Ética em Pesquisa com Seres Humanos da UFTM pelo protocolo $n$. o $2188 / 2011$.

As variáveis de interesse foram: Influências na sexualidade relacionada à religião, pais ou responsáveis, universidade e ingestão de bebidas alcoólicas; Satisfação com aspectos da vida acadêmica, da vida pessoal e com suas interações sociais. Todas as informações coletadas foram armazenadas em um banco de dados construído no software de domínio público 
Excel v.2007 (Microsoft Corporation, Estados Unidos). A partir dos resultados, procedeu-se análise descritiva por frequências absolutas (n) e percentuais (\%) para as variáveis categóricas, e medidas de centralidade e de dispersão para as variáveis numéricas.

\section{RESULTADOS}

Fizeram parte do estudo 786 alunos, dos quais $623(79,3 \%)$ eram do sexo feminino e $163(20,7 \%)$ do sexo masculino. A idade média encontrada foi de 19,2 $\pm 3,1$ anos, a maioria se declarava branco $(64,6 \%)$, seguido de pardo $(31,9 \%)$ e negro $(3,4 \%)$. Cerca de $67,0 \%$ não possuiam religião e 46,6\% eram procedentes do Estado de São Paulo, enquanto Minas Gerais respondia por $41,5 \%$ dos estudantes. $61,5 \%$ possuíam renda familiar acima de três a cinco salários

Tabela 1 - Acadêmicos UFTM quanto influências na sexualidade. Uberaba, 2012.

\begin{tabular}{|c|c|c|c|c|c|c|c|}
\hline & & \multicolumn{2}{|c|}{ Feminino } & \multicolumn{2}{|c|}{ Masculino } & \multicolumn{2}{|c|}{ Total } \\
\hline & & $\mathbf{n}$ & $\%$ & $\mathbf{n}$ & $\%$ & $\mathbf{n}$ & $\%$ \\
\hline \multicolumn{8}{|c|}{ Possíveis influências na Sexualidade } \\
\hline \multirow[t]{4}{*}{ Religião } & Reprime & 214 & 34,3 & 87 & 53,4 & 301 & 38,3 \\
\hline & Nenhuma & 220 & 35,3 & 36 & 22,1 & 256 & 32,6 \\
\hline & Estimula & 126 & 20,2 & 21 & 12,9 & 147 & 18,7 \\
\hline & Orienta & 63 & 10,1 & 19 & 11,7 & 82 & 10,4 \\
\hline \multirow[t]{4}{*}{ Pais/responsáveis } & Estimula & 225 & 36,1 & 48 & 29,4 & 273 & 34,7 \\
\hline & Orienta & 157 & 25,2 & 52 & 31,9 & 209 & 26,6 \\
\hline & Reprime & 157 & 25,2 & 26 & 16,0 & 183 & 23,3 \\
\hline & Nenhuma & 84 & 13,5 & 37 & 22,7 & 121 & 15,4 \\
\hline \multirow[t]{4}{*}{ Universidade } & Estimula & 318 & 51,0 & 77 & 47,2 & 395 & 50,3 \\
\hline & Orienta & 220 & 35,3 & 42 & 25,8 & 262 & 33,3 \\
\hline & Nenhuma & 75 & 12,0 & 29 & 17,8 & 104 & 13,2 \\
\hline & Reprime & 10 & 1,6 & 15 & 9,2 & 25 & 3,2 \\
\hline \multirow{5}{*}{$\begin{array}{l}\text { Ingestão de bebida } \\
\text { alcoólica }\end{array}$} & Estimula & 605 & 97,1 & 154 & 94,5 & 759 & 96,6 \\
\hline & Nenhuma & 9 & 1,4 & 6 & 3,7 & 15 & 1,9 \\
\hline & Orienta & 6 & 1,0 & 2 & 1,2 & 8 & 1,0 \\
\hline & Reprime & 3 & 0,5 & 1 & 0,6 & 4 & 0,5 \\
\hline & Total & 623 & 79,3 & 163 & 20,7 & 786 & 100,0 \\
\hline
\end{tabular}

Na Tabela 2 são apresentados os resultados sobre a satisfação dos acadêmicos com a universidade, com a vida pessoal e suas interações sociais. A maioria dos jovens $(54,3 \%)$, sobretudo os do sexo masculino mínimos (41,7\%), estabelecido à época da pesquisa em $\mathrm{R} \$ 622,00$. A maioria dos adolescentes, tanto do sexo feminino $(57,9 \% ; 361)$ quanto do sexo masculino (50,9\%; 83) mora com amigos e cerca de $70,0 \%$ das mulheres e $60,7 \%$ dos homens residem em pensionatos ou repúblicas.

Na tabela 1, apresentam-se os dados aferidos das percepções dos jovens quanto à influência de algumas variáveis em sua sexualidade. Para grande parte dos jovens (34,3\% das mulheres e $53,4 \%$ dos homens) a religião tem efeito repressor. As demais variáveis apresentam efeitos de estímulo na sexualidade dos jovens estudados $(34,7 \%$ no caso dos pais, $50,3 \%$ universidade e $96,6 \%$ consumo de bebida alcoólica), com flutuações proporcionais e distribuição desigual entre os sexos estudados.

$(71,8 \%)$ se dizem satisfeitos com a Universidade. Em relação aos aspectos da vida pessoal, $58,1 \%$ das mulheres e $89,0 \%$ dos homens estavam insatisfeitos ou tinham respostas ambíguas; proporções ainda mais 
acentuadas nos parâmetros de interação social, em que cerca de um quarto dos adolescentes $(26,0 \%)$ se consideravam felizes.

Tabela 2 - Acadêmicos UFTM quanto à satisfação com aspectos de vida. Uberaba, 2012.

\begin{tabular}{|c|c|c|c|c|c|c|c|}
\hline & & \multicolumn{2}{|c|}{ Feminino } & \multicolumn{2}{|c|}{ Masculino } & \multicolumn{2}{|c|}{ Total } \\
\hline & & $\mathbf{n}$ & $\%$ & $\mathbf{n}$ & $\%$ & $\mathbf{n}$ & $\%$ \\
\hline \multicolumn{8}{|c|}{ Satisfação com aspectos da vida } \\
\hline \multirow[t]{3}{*}{ Vida Acadêmica } & Satisfeito & 310 & 49,8 & 117 & 71,8 & 427 & 54,3 \\
\hline & Indiferente & 220 & 35,3 & 40 & 24,5 & 260 & 33,1 \\
\hline & Insatisfeito & 93 & 14,9 & 6 & 3,7 & 99 & 12,6 \\
\hline \multirow[t]{3}{*}{ Vida Pessoal } & Indiferente & 276 & 44,3 & 88 & 54,0 & 364 & 46,3 \\
\hline & Satisfeito & 261 & 41,9 & 18 & 11,0 & 279 & 35,5 \\
\hline & Insatisfeito & 86 & 13,8 & 57 & 35,0 & 143 & 18,2 \\
\hline \multirow[t]{4}{*}{ Interações sociais } & Indiferente & 255 & 41,1 & 73 & 44,8 & 328 & 41,8 \\
\hline & Insatisfeito & 207 & 33,3 & 45 & 27,6 & 252 & 32,1 \\
\hline & Satisfeito & 161 & 25,6 & 45 & 27,6 & 204 & 26,0 \\
\hline & Total & 623 & 79,3 & 163 & 20,7 & 786 & 100,0 \\
\hline
\end{tabular}

\section{DISCUSSÃO}

A população estudada inclui apenas alunos do primeiro semestre de cursos universitários da área de saúde, que se constitui em uma população ainda pouco estudada e que apresenta características peculiares em âmbito emocional, psicológico, experiência de vida e outros.

Entendendo o momento de ingresso na Universidade como um acontecimento importante na vida do jovem, considera-se a hipótese de que os mesmos estejam abertos a novas experiências, influenciados por diversas alterações em sua rotina, como a experiência de morar longe dos pais e /ou com amigos e colegas da universidade, a grande disponibilidade de eventos noturnos e festas que envolvem o consumo de bebidas alcóolicas, e outras situações, pelas quais poderiam estar mais susceptíveis a manifestarem sua sexualidade. Dos pesquisados $67,6 \%$ consideraram sua atividade de lazer preferida estar presente em festas ou bailes.

Em estudo que investigou o perfil de alunos ingressantes no âmbito da saúde em uma universidade pública do interior de Minas Gerais, destacou que entre os 124 participantes, a maioria pertencia ao sexo feminino (89,0\%), com 18 anos (34,0\%), de cor branca $(81,5 \%)$, procedente de São Paulo
$(50,8 \%)$, com renda familiar de até quatro salários $(19,4 \%)$. Em relação ao perfil de saúde, $35,5 \%$ não praticavam atividade física, $2,4 \%$ eram fumantes, $31,5 \%$ consumiam bebida alcoólica e 45,2\% possuíam vida sexual ativa ${ }^{4}$.

Com relação às possíveis influências de aspectos da vida do adolescente à manifestação de sua sexualidade, as maiores proporções encontradas foram: influência de estímulo pelo uso de bebidas alcóolicas $(96,6 \%)$, ingresso na universidade $(50,3 \%)$ e de pais ou responsáveis $(34,7 \%)$; além de repressão por parte da religião $(38,3 \%)$. Ressalta-se que apesar de não ser objeto deste estudo investigar qual a crença religiosa dos indivíduos, a variável referente a possuir religião e qual sua vertente pode estar relacionada à sexualidade.

Por esta linha, estudo realizado com 395 universitários mexicanos, identificou uma relação maior com a manutenção da virgindade e iniciação sexual mais tardia com influencia religiosa, sobretudo entre católicos ${ }^{10}$.

Estudo que incluiu adolescentes das 27 capitais brasileiras encontrou associação positiva ( $p>0.001)$ entre os adolescentes que praticaram relações sexuais sem uso de camisinha e consumo de álcool e drogas ilícitas, cujas proporções foram 32,0\% e 
$43,0 \%$ respectivamente maiores, a considerar o uso nos últimos 30 dias anteriores à pesquisa ${ }^{11}$. Ainda neste aspecto, estudo que examinou a frequência de uso de álcool na adolescência como preditor do comportamento sexual de risco para HIV na idade adulta, encontrou probabilidade de 1.78 vezes maior chance (IC95\%, 1,05-3,02) de risco para aqueles que consumiam álcool frequentemente, sendo este um importante marcador para a elaboração de programas que visem prevenir comportamento sexual de risco ${ }^{12}$.

Os ingressantes na universidade são inseridos em contextos adversos e situações de crises que requerem processos de adaptação, podendo desencadear o aparecimento de estados de depressão, alcoolismo, evasão escolar, dificuldades na aprendizagem, nos relacionamentos pessoais e isolamento social ${ }^{13,14}$. Tal condição pode gerar dificuldades no convívio e na interação social dos respondentes desta pesquisa, uma vez que apenas $26,0 \%$ dos estudantes estavam satisfeitos com seus relacionamentos e 35,5\% com os parâmetros adotados para avaliar sua vida pessoal.

Dos pesquisados, 54,3\% responderam estar satisfeitos com a universidade. Refletese que a identificação pessoal com o curso escolhido, sobretudo nos períodos iniciais da graduação, é considerada uma importante fonte de satisfação, tendo em vista que o comprometimento com uma escolha em termos vocacionais encerra uma avaliação mais otimista das possibilidades, relativiza as dificuldades eventuais para obtenção de resultados e promove um maior bem-estar psicológico, até mesmo em outros parâmetros da vida pessoal ${ }^{15}$.

Estudo que incluiu uma população de universitários em distintos níveis de formação identificou bons níveis de satisfação de vida, com curso e profissão, sendo que os alunos em estágios iniciais de formação apresentam maior comprometimento, mas menor exploração vocacional que os demais estudantes ${ }^{3}$.
Estudo realizado com acadêmicos de uma universidade do Espírito Santo, identificou indices de satisfação com o curso de $89,4 \%$, associada com o fato de terem aprovação no último semestre $(89,6 \%)$. Além disso, no mesmo estudo, verificou-se alguns fatores que influenciavam no consumo de substâncias psicoativas (lícitas e ilícitas), encontrando associações positivas com o acesso a locais alheios à universidade e à opção de faltar às aulas para dormir ou descansar ${ }^{16}$.

Tais fatos denotam que o papel da universidade não contempla apenas a perspectiva intramuros, mas deve ater-se às questões inerentes aos distintos aspectos da vida de seus estudantes.

Assim, questiona-se a influência da universidade neste contexto, tanto quanto as implicações das condutas de saúde dos estudantes em sua prática futura. Fatores como a percepção de invulnerabilidade e influências em condutas de risco praticadas por estes jovens - como o consumo indiscriminado de bebidas alcoólicas em ocasiões comemorativas no contexto universitário - refletem a necessidade de um trabalho extenso na formação dos futuros profissionais do campo da saúde.

\section{CONCLUSÃO}

Os achados da presente investigação apontam um perfil de universitários predominantemente do sexo feminino, com idade média de 19,2 $\pm 3,1$ anos, brancos, sem religião, procedentes do Estado de São Paulo seguido de Minas Gerais, com renda familiar entre três e cinco salários mínimos.

A maioria dos jovens morava com amigos, em pensionatos ou repúblicas e tinham como atividade de lazer preferida o ato de ir a festas ou bailes noturnos. Grande parte dos jovens estava satisfeita com a universidade e pouco satisfeitos com suas interações sociais e acreditava que o fato de estarem na universidade e consumir bebidas alcoólicas pode influenciar sua sexualidade.

Os dados, apesar de refletirem características de uma única intituição de 
ensino superior, chamam a atenção para questões elementares que podem significar falhas nos processos iniciais da formação de profissionais da saúde. Recomenda-se, desta forma, que ações de educação em saúde sejam direcionadas aos adolescentes que adentram as universidades, sobretudo os da área de saúde, preparando esses futuros profissionais tanto para uma vida mais saudável, destacando não somente aspectos biológicos, mas também psicossociais e comportamentais.

A vulnerabilidade desses jovens deve ser enfatizada, não apenas no aspecto individual, mas também no programático e no social, contribuindo para a formação de alunos críticos, visando à transformação da realidade.

\section{REFERÊNCIAS}

1. Silveira RE, Santos AS. Contextos de vulnerabilidade entre adolescentes do ensino fundamental de Uberaba/MG. Enferm Foco (Brasília). 2012; 3(4):182-5.

2. Aquino PS, Brito FEV. Perfil sexual de adolescentes universitários de um curso de graduação em enfermagem [Internet]. REM Rev Min Enferm. 2012 [citado em 14/08/13]; 16(3):324-9. Disponível em: http://www.dx.doi.org/S1415-

27622012000300003.

3. Bardagi MP, Hutz CS. Undergraduate students' life satisfaction, career commitment and career exploration. Arq Bras Psicol. 2010; 62(1):159-70. 4. Nardelli GG, Gaudenci EM, Garcia BB, Carleto CT, Gontijo LM, Pedrosa LAK. Perfil dos alunos ingressantes dos cursos da área da saúde de uma universidade federal. REAS. 2013; 2(1):3-12.

5. Dessunti EM, Reis AOA. Vulnerabilidade às DST/AIDS entre estudantes da saúde: estudo comparativo entre primeira e última série. Cienc Cuid Saude. 2012; 11(suplem):274-83.

6. Teva I, Bermudez M, Ramiro M, Buela-Casal G. Current epidemiological situation of HIV/AIDS in Latin America: analysis of differences among countries[Internet]. Rev Med Chil. 2012 [citado em citado em 19/09/13]; 140(1):50-8. Disponível em: http:// http://www.scielo.cl/pdf/rmc/v140n1/art07.pd f. doi:10.4067/S0034-98872012000100007.
7. Barbosa RG, Garcia FCP, Manzato AJ, Martins RA, Vieira FT. Conhecimento sobre DST/AIDS, hepatites e conduta sexual de universitários de São José do Rio Preto, SP. DST J Bras Doenças Sex Transm. 2006; 18(4):224-30.

8. Leite MTF, Costa AVS, Carvalho KACC, Melo RLR, Nunes BMTV, Nogueira LT. Saber e prática contraceptiva e prevenção de DST/HIV/AIDS em universitários da área da saúde. Rev Bras Enferm. 2007; 60(4):434-8.

9. Alves AS, Lopes MHBM. Use of contraceptive methods among university adolescents. Rev Bras Enferm. 2008; 61(2):170-7.

10. Moral-De la Rubia J. Religion, meanings and attitudes toward sexuality: a psychosocial approach. Rev Colomb Psicol. 2010; 19(1):45-59. 12. Muchimba M, Haberstick BC, Corley RP, McQueen MB. Frequency of alcohol use in adolescence as a marker for subsequent sexual risk behavior in adulthood. J Adolesc Health. 2013. doi:10.1016/j.jadohealth.2013.02.005.

13. Eurich RB, Kluthcovsky ACGC. Evaluation of quality of life of undergraduate nursing students from first and fourth years: the influence of sociodemographic variables. Rev. Psiquiatr Rio Gd Sul. 2008; 30(3):211-20.

14. Sanchez ZM, Nappo SA, Cruz JI, Carlini EA, Carlini CM, Martins SS. Sexual behavior among high school students in Brazil: alcohol consumption and legal and illegal drug use associated with unprotected sex. Clinics (São Paulo). 2013; 68(4):489-94.

15. Alves AS, Lopes MHBM. Locus of control and contraceptive knowledge, attitude and practice among university students. Rev Saúde Pública. 2010; 44(1):39-44.

16. Portugal $S Q$, Siqueira MM. Fatores associados ao uso de substâncias psicoativas entre universitários de pedagogia da universidade federal do Espírito Santo. Cad Saúde Coletiva 2011; 19(3):348-55.

CONTRIBUIÇÕES
Rodrigo Eurípedes da Silveira participou
no desenho do estudo, na coleta e análise dos
dados e na redação do artigo;
Álvaro da Silva Santos atuou no desenho do
estudo, na revisão crítica e na redação do
artigo;
Maritza Rodrigues Borges teve
participação na coleta de dados e redação do
artigo.

